\title{
Getting comfortable hand configurations while manipulating an object
}

\author{
Andrés Montaño and Raúl Suárez \\ Institute of Industrial and Control Engineering (IOC) \\ Universitat Politècnica de Catalunya (UPC) - Barcelona Tech \\ Barcelona, Spain, \{andres.felipe.montano, raul.suarez\}@upc.edu
}

\begin{abstract}
The paper presents an approach to manipulate unknown objects based on tactile information and force feedback. The object manipulation is performed using two fingers of the Shunck Dexterous Hand, which is equipped with tactile sensors on the fingertips. The contact point on each fingertip is modeled using a virtual articulation which adds a virtual degree of freedom to the finger. The approach uses the tactile data and hand kinematics information in order to estimate a grasp quality measure and to make finger adjustments after an initial grasp in order to improve the hand comfort. The approach was implemented in a real sensorized hand, and some examples manipulating different objects are presented in the paper showing the evolution of the resulting quality.
\end{abstract}

\section{INTRODUCTION}

Dexterous manipulation is the capability to change the position and orientation of an object with respect to the hand while keeping a stable grasp [1]. Dexterous manipulation is closely related with the development of grasping elements, some of them with anthropomorphic characteristics [2], [3], [4], [5], [6]. Tactile information is important for robotic hands in order to achieve dexterity and precise object handling.

A task example that requires dexterous manipulation could be the opening and closing of the screw cap of a bottle using the fingers without changing the wrist position. Usually, during the object manipulation, it is expected that contact points between the hand and the object are located in specific locations. However, in more complex applications the location and nature of the contact points can not be precisely predicted or can not be modeled in advance [7].

Object manipulation using robotic hands equipped with tactile sensors to detect contacts and increase their capabilities is a challenging subject. This paper presents an approach to manipulate unknown objects based on tactile information and force feedback.

Robot grasping and manipulation require very accurate knowledge of the location of the object within the robotic hand. A vision system could not provide very precise and robust pose tracking due to occlusions or light limitations. For this reason, visual information has been combined with kinematics and tactile information in order to estimate the pose of a grasped object [8]. The tactile information can be treated as a sequence of images in order to extract information about the contact conditions between an object and the hand [9],

This work was partially supported by the Spanish Government through the projects DPI2010-15446, DPI2011-22471 and DPI2013-40882-P.

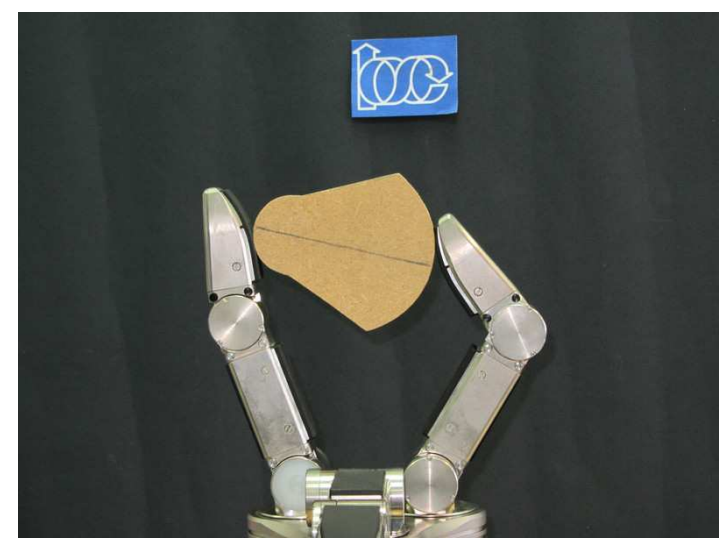

Fig. 1. The Schunk Dexterous Hand (SDH2) manipulating an unknown object using 2 fingers with tactile feedback.

and therefore image processing techniques are used to process the tactile sensor information. On the other hand, machine learning techniques have been also applied in order to improve object manipulation using tactile information, specifically, in order to estimate the grasp stability [10], [11]. In a previous work [12] the shape of an unknown object was recognized using manipulation and tactile information obtained during handling.

As a difference with most of the previous works commented above, in this work the dexterous manipulation is performed without previous information about the grasped object, the tactile information provided by the sensors is combined with the hand kinematic information in order to estimate the grasp quality. The changes in the grasp quality define the movements of the fingers in order to manipulate the unknown object, i.e. the manipulation strategy is defined considering the grasp quality to change the grasp pose in order to improve the comfort of the hand. A grasp is considered comfortable if the hand joints are close to the center of their ranges, which also allows a wide set of future movements. Thereby, a manipulation task can be completed using this wide set of movements. Besides, the paper presents a kinematics model of the robotic hand that includes the tactile sensor on the fingertips. The model uses a virtual link in order to include the contact point information, this virtual link adds an extra dof to each finger.

The remainder of the paper is organized as follows. After this introduction, Section II introduces the bases of the proposed approach. In Section III descriptions of the hand 
kinematics and the friction constraints are presented. The bases of the object manipulation approach are discussed in Section IV. Experimental results are described in Section V. Finally, Section VI presents the conclusions and future work.

\section{APPROACH OVERVIEW}

The robotic hand used in this work is the Schunk Dexterous Hand (SDH2) shown in Figure 1. This is a three finger hand with seven active degrees of freedom (dof). The SDH2 has tactile sensors attached to the surfaces of the proximal links and the distal links (fingertips), thus the tactile sensor system has six sensor pads. Two fingers of the hand are coupled and can be rotated on the base to work opposite to each other in the same plane (see Figure 1). To manipulate an unknown object, the fingertips of the two coupled fingers of the robotic hand are used to perform a prismatic precision grasp [13], which is comparable with a human grasp using the thumb and index finger, thus only the two sensor pads of the fingertips of the coupled fingers are used. The manipulation strategy must guarantee hand configurations which change the relative pose between the object and the hand avoiding object falls, and also improve the comfort of the hand.

Each fingertip tactile sensor of the hand gives information about the relative position of the contact point with respect to the sensor frame, and about the force applied on the object by the fingertip. Once the object is grasped, the position of the contact points, the distance between the contact points on each fingertip, the contact force, and the grasp quality, are computed using the contact information of both tactile sensors. This information will be used to determine the proper object manipulation, which must avoid the object falls and look for a comfortable hand configuration.

After each manipulation step, the fingers are relocated and as a consequence the grasp quality measure is modified. One of the objectives of the proposed approach is to improve the grasp quality via the object manipulation. The proposed manipulation strategy searches for the movements of the hand that improve the grasp quality in each manipulation step. Consider a search space defined by the position of the finger joints. The movement direction that improves the grasp quality is defined by the gradient of the grasp quality function (ascendant or descent depending on the case). Figure 2 shows a virtual example of the contours of a grasp quality measure for a two $\operatorname{dof}\left(\theta_{1}\right.$ and $\left.\theta_{2}\right)$ robot hand. Quality is represented by concentric circles, it increases from the external contour towards the more centric contour. The black line represents the configurations of the hand during an object manipulation, in this example the manipulation starts at the configuration determined by the point $(0,50)$ and it evolves to the point $(50,50)$. The first five hand configurations are around the same contour so they have approximately the same quality, and then the following hand configurations move to inner contours meaning an improvement in the grasp quality.

\section{HAND KINEMATICS}

The tactile sensor pad on each proximal link of the SDH2 has $84(6 \times 14)$ tactile sensor cells of $3.4 \mathrm{~mm} \times 3.4 \mathrm{~mm}$ while the pad over each fingertip has $70(4 \times 4+6 \times 9)$ cells. When the fingertips touch an object the contact is in general produced

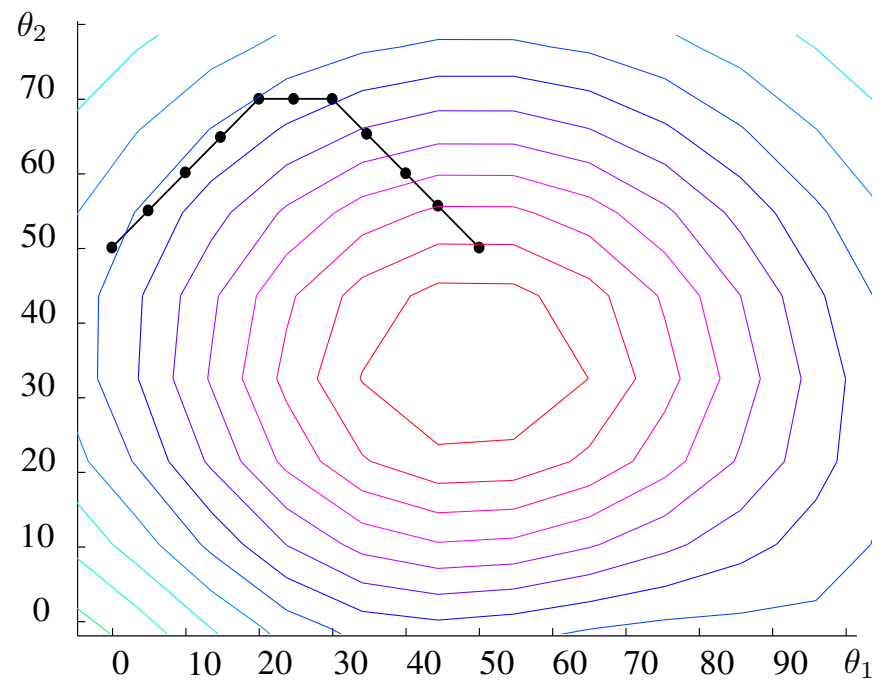

Fig. 2. Virtual example of the contours of a grasp quality function.

\begin{tabular}{c|c|c|c|c}
\hline Joint. & $\alpha_{i}$ & $a_{i}$ & $d_{i}$ & $\theta_{i}$ \\
\hline 1 & 90 & 0 & 0 & $\theta_{1}$ \\
2 & 0 & 86.5 & 0 & $\theta_{2}+\pi / 2$ \\
3 & 0 & 68 & 0 & $\theta_{3}+\pi / 2-\varphi_{1}$ \\
4 & 0 & $r_{1}$ & 0 & 0 \\
\hline
\end{tabular}

TABLE I. DENAVIT-HARTENBERG PARAMETERS FOR FINGER $f_{1}$ OF THE SDH2.

on a contact area, in this work the contacts are considered to be punctual and the contact points are considered to be located at the barycenter of the contact area on the sensor pad. Thus, the two contacts are represented by the points $P_{1}$ on finger $f_{1}$ and $P_{2}$ on finger $f_{2}$.

\section{A. Direct Kinematics}

The aim of the direct kinematics is to find the position of a potential contact point $P_{1}$ given the joints values $\theta_{2}$ and $\theta_{3}$ considering the shape of the fingertip and the geometry of the finger. Denavit-Hartenberg parameters [14] are used to describe the kinematic model of the fingers. Each finger has a reference system at the finger base. Figure $3 \mathrm{a}$ shows the reference systems used to compute the DH-parameters for the finger $f_{1}$. The distance between the reference systems of the fingers $f_{1}$ and $f_{2}$ is $66 \mathrm{~mm}$ along axis- $x_{0}$. The reference system of the finger $f_{1}$ is the global reference system. Table I shows the DH-parameters for finger $f_{1}$, where joint 4 refers to a virtual link from the last finger joint to the contact point. The reasoning for the finger $f_{2}$ is the same as the described for finger $f_{1}$. The range of all the joints excepting the coupled joint is from -90 to +90 degrees (See Figure 4). For the coupled joints the range is from 0 to 90 degrees, being 90 degrees the used value to work with the finger is an opposite way.

Since the tactile sensor of each fingertip is not planar, the shape of the sensor needs to be considered in order to compute the position of the potential contact point in the absolute reference frame. The sensor surface is composed of two parts, an arc of a circle with radius $60 \mathrm{~mm}$ centered at point $K=(33.5,-45)$ in the reference system $x_{3} y_{3}$, and a segment of a straight line as seen in Fig. 3b. The processing of a sensor 

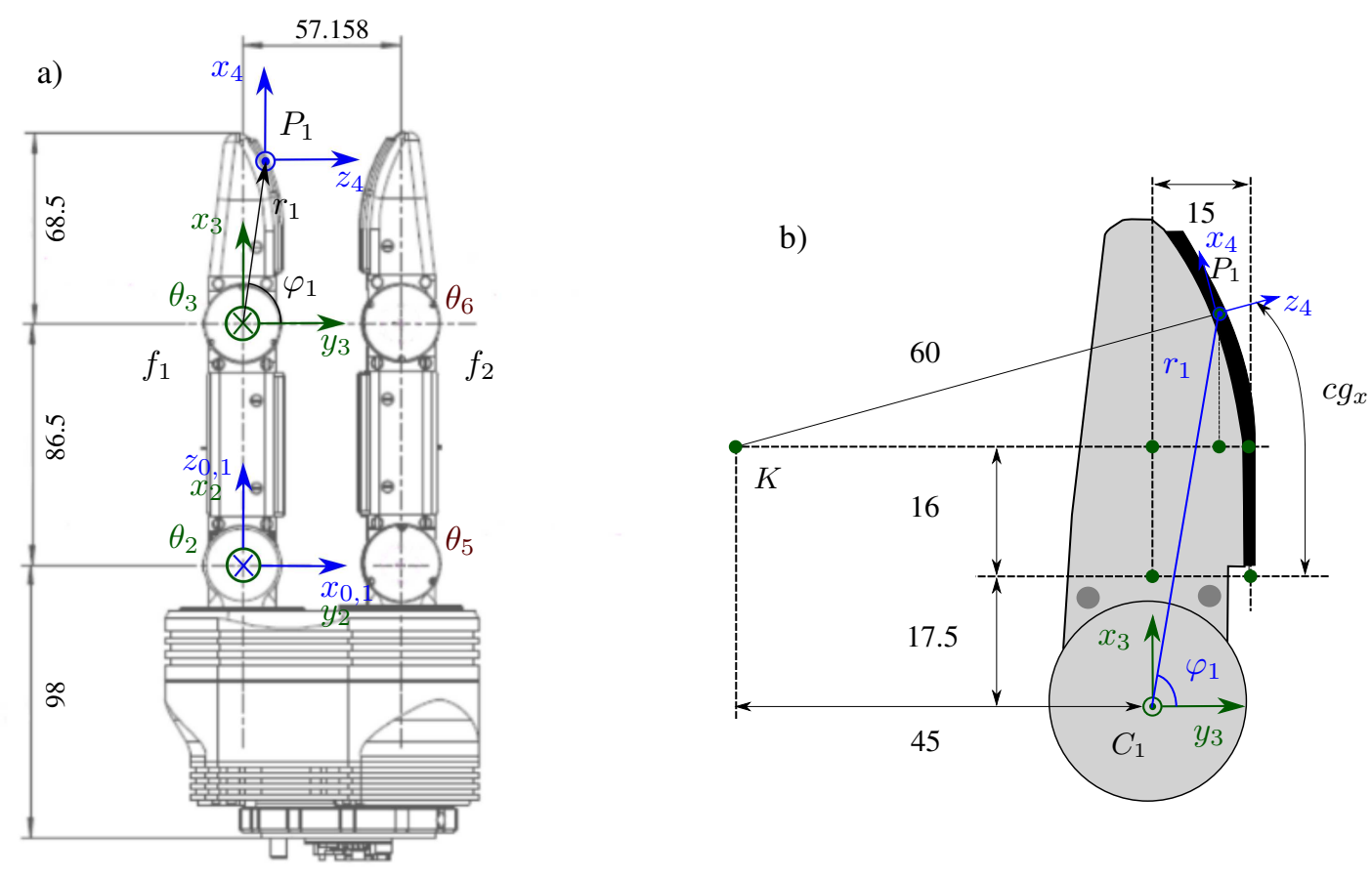

Fig. 3. Dimensions and coordinate systems on SDH2 used for kinematics analysis. a) Front view. b) Zoom of the fingertip.

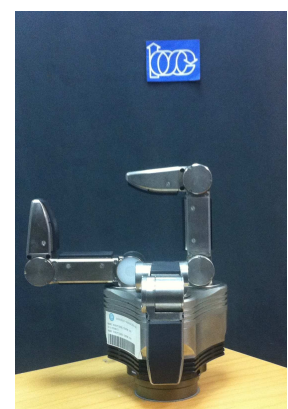

a)

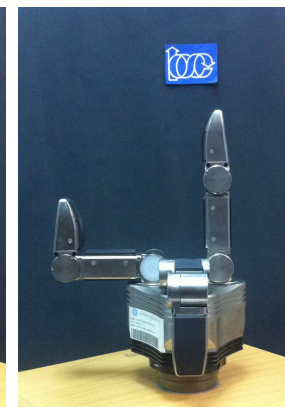

b)

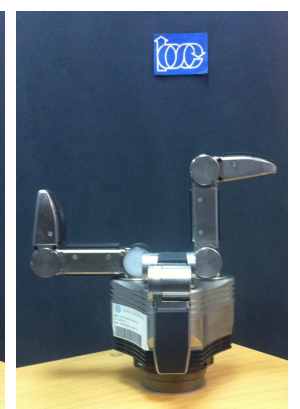

c)
Fig. 4. Finger distal joint of the SDH2 in different configurations. a) 90 degrees. b) 0 degrees. c) -90 degrees.

measurement during contact gives the position $\left(c g_{x}, c g_{y}\right)$ of the barycenter of the contact area in the sensor reference system. In this work only the $x$ component, $c g_{x}$, is considered since the manipulation is performed in the plane $x_{0} z_{0}$ and the $y$ component, $c g_{y}$, does not add relevant information. Given $c g_{x}$, the coordinates of the contact point $P_{1}$ in the reference system $x_{3} y_{3}$ are given by,

$$
\begin{gathered}
P_{1_{x}}= \begin{cases}17.5+c g_{x} & \text { if } c g_{x}<16 \\
33.5+60 \sin \left(\frac{c g_{x}-16}{60}\right) & \text { if } c g_{x} \geq 16\end{cases} \\
P_{1_{y}}= \begin{cases}15 & \text { if } 17.5<P_{1_{x}}<33.5 \\
-45+\sqrt{60^{2}-\left(P_{1_{x}}-33.5\right)^{2}} & \text { if } 33.5 \leq P_{1_{x}}<66.4\end{cases}
\end{gathered}
$$

As mentioned above, a virtual link is used in order to include the contact point information. This virtual link adds an extra $d o f$ to the finger. The reference system $x_{4} y_{4}$ is added at the barycenter of the contact area. The distance $r_{1}$ between the origins of the reference systems $x_{3} y_{3}$ and $x_{4} y_{4}$, and the angle $\varphi_{1}$ between the $y_{3}$-axis and virtual link described by $r_{1}$ are used to include the contact point information. $r_{1}$ and $\varphi_{1}$, referenced to the $x_{3} y_{3}$ frame, are given by,

$$
\begin{aligned}
& r_{1}=\sqrt{{P_{1_{x}}{ }^{2}+P_{1_{y}}{ }^{2}}_{\varphi_{1}}=\arctan \left(\frac{P_{1_{x}}}{P_{1_{y}}}\right)}
\end{aligned}
$$

The position of the contact point $P_{1}$ and the point $C_{1}$ the origin of the frame $x_{3} y_{3}$, referenced to the finger frame, can be computed using the values of $r_{1}$ and $\varphi_{1}$, and the joint values $\theta_{2}$ and $\theta_{3}$ as,

$$
\begin{gathered}
P_{1}=\left[\begin{array}{c}
86.5 s \theta_{2}+r_{1} c \theta_{2} c\left(\theta_{3}-\varphi_{1}\right)-s \theta_{2} s\left(\theta_{3}-\varphi_{1}\right) \\
0 \\
86.5 c \theta_{2}+r_{1}\left(-c\left(\theta_{3}-\varphi_{1}\right) s \theta_{2}-c \theta_{2} s\left(\theta_{3}-\varphi_{1}\right)\right.
\end{array}\right] \\
C_{1}=\left[\begin{array}{c}
86.5 s \theta_{2} \\
0 \\
86.5 c \theta_{2}
\end{array}\right]
\end{gathered}
$$

\section{B. Inverse Kinematics}

The inverse kinematic problem appears when, given the absolute position of the contact point $P_{1}, r_{1}$ and, $\varphi_{1}$, it is necessary to find the values of $\theta_{2}$ and $\theta_{3}$ to properly contact at $P_{1}$ (the same reasoning is applied to finger $f_{2}$ ). There are different approaches to compute the inverse kinematics, in this work we use a geometric approach since the manipulation is done on a plane and using two dof per finger. Figure 5 shows the geometric parameters used to solve the inverse kinematics 


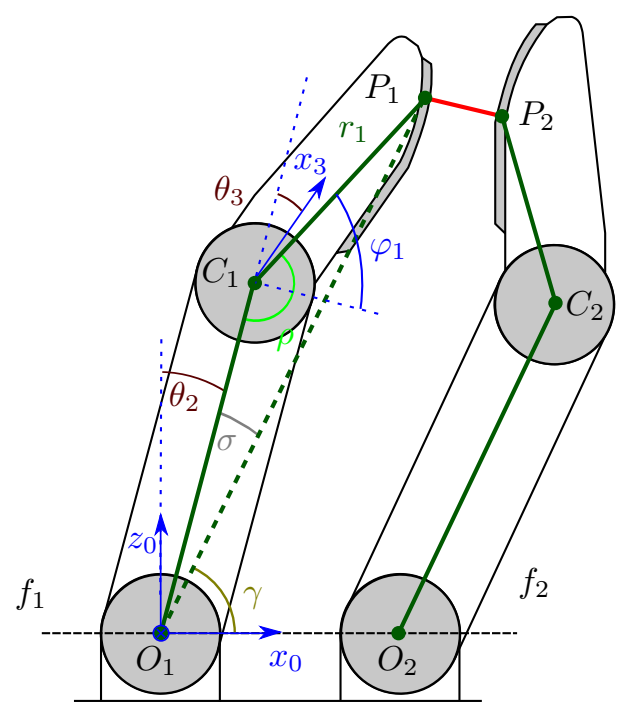

Fig. 5. Two fingers model of the SDH2 used to compute inverse kinematics.

problem. The values of the angles $\rho, \sigma$ and $\gamma$ are computed using the cosine law as,

$$
\begin{gathered}
\rho=\arccos \left(\frac{-\left|O_{1} P_{1}\right|^{2}+\left|O_{1} C_{1}\right|^{2}+\left|C_{1} P_{1}\right|^{2}}{2\left|O_{1} C_{1}\right|\left|C_{1} P_{1}\right|}\right) \\
\sigma=\arccos \left(\frac{-\left|C_{1} P_{1}\right|^{2}+\left|O_{1} C_{1}\right|^{2}+\left|O_{1} P_{1}\right|^{2}}{2\left|O_{1} C_{1}\right|\left|O_{1} P_{1}\right|}\right) \\
\gamma=\arctan \left(\frac{P_{1_{z}}}{P_{1_{x}}}\right)
\end{gathered}
$$

There are two possible solutions for a given $P_{1}$, however, considering the geometric constraints imposed by the manipulation problem where the desired configuration belongs to the section workspace which permits that the finger works opposed to the other finger, thus, valid configurations are obtained only for values of $\theta_{3}$ satisfying $\theta_{3}>\varphi_{1}-\pi / 2$. Then, the values for $\theta_{2}$ and $\theta_{3}$ are given by,

$$
\begin{gathered}
\theta_{2}=-\sigma-\gamma+\operatorname{sign}\left(P_{1_{x}}\right) \pi / 2 \\
\theta_{3}=\rho-\pi / 2-\varphi_{1}
\end{gathered}
$$

where

$$
\operatorname{sign}(x)= \begin{cases}1 & \text { if } x \geq 0 \\ -1 & \text { if } x<0\end{cases}
$$

\section{Friction Constraints}

In order to avoid sliding, each force applied on the object must be located within the friction cone centered at the direction normal to the object surface at the contact point. A planar grasp with two frictional contact points is force-closure when the segment connecting the contact points lies inside the friction cone at both contact points (see Figure 6). The friction cone is given by $\alpha=\arctan \mu$, with $\mu$ being the friction coefficient (Coulomb friction model). Any applied force that belongs to the friction cone will not produce slippage, therefore the angle $\beta_{i} i=1,2$, between the normal direction at each contact point and the segment between the two contact points

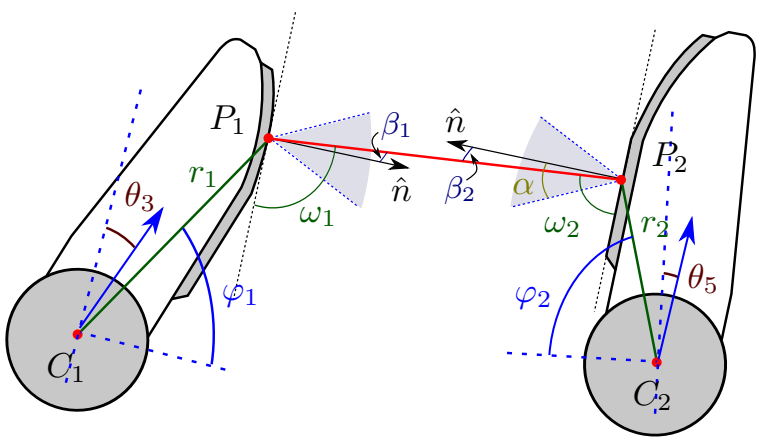

Fig. 6. Detail of the fingertips and angles considered to compute the friction constraints.

must satisfy $\beta_{i}<\alpha$. Then, the above condition can be expressed as,

$$
\pi / 2-\alpha<\omega_{i}<\pi / 2+\alpha
$$

where $\psi_{i}, i=1,2$ is computed for both contact points as,

$$
\begin{aligned}
& \psi_{1}=\arccos \left(\frac{-\left|C_{1} P_{2}\right|^{2}+r_{1}^{2}+\left|P_{1} P_{2}\right|^{2}}{2 r_{1}\left|P_{1} P_{2}\right|}\right)-\theta_{3}-\pi / 2+\varphi_{1} \\
& \psi_{2}=\arccos \left(\frac{-\left|C_{2} P_{1}\right|^{2}+r_{2}^{2}+\left|P_{1} P_{2}\right|^{2}}{2 r_{2}\left|P_{1} P_{2}\right|}\right)-\theta_{5}-\pi / 2+\varphi_{2}
\end{aligned}
$$

\section{OBject Manipulation}

To initially grasp an object, the fingers start their movements from a wide open position and they are closed over the object until the desired $F^{d}$ is reached. The fingers perform a prismatic precision grasp using the fingertips. So, the initial contact points on the object are unknown and they change in each different execution of the manipulation process for the same object. Once the initial grasp is performed, the initial contact points are computed using the contact information as described in the previous section, and they are used as the initial conditions for the manipulation process. The grasping force $F_{k}$ is computed as the average of the contact forces $F_{1_{k}}$ and $F_{2_{k}}$ measured by the sensors of both fingertips,

$$
F_{k}=\frac{F_{1_{k}}+F_{2_{k}}}{2}
$$

where $k$ denotes a manipulation step.

Another important variable of the contact information is the distance $d_{k}$ between the contact points $P_{1_{k}}$ and $P_{2_{k}}$, which is given by,

$$
d_{k}=\sqrt{\left(P_{1_{k_{x}}}-P_{2_{k_{x}}}\right)^{2}+\left(P_{1_{k_{z}}}-P_{2_{k_{z}}}\right)^{2}}
$$

Different quality metrics can be used to value a grasp configuration. In this work we consider a comfort quality index, $Q_{c}$, to evaluate the grasp quality during the manipulation process. This quality index, associated with the hand configuration, favors the hand configurations with the finger joints as far as possible from their physical limits, i.e. with the joint positions as close as possible to the center of their ranges [15]. A complete survey on grasp qualities measures can be found in [16], [17]. The used comfort quality index $Q_{c}$ is given by,

$$
Q_{c}=\sum_{i=1}^{n m}\left(\frac{\theta_{i}-\theta_{0 i}}{\theta_{\max _{i}}-\theta_{\min _{i}}}\right)^{2}
$$


where $\theta_{i}$ and $\theta_{0 i}$ are the actual and the middle-range positions of the i-th joint, respectively. The minimization of $Q_{c}$ implies a grasp configuration with the joint positions close to the middlerange reference position.

Algorithm 1 summarizes the procedure used to manipulate an object using tactile feedback. It requires as input the desired force $F^{d}$ to be applied to the grasped object. First, the fingers are closed until the contact force $F_{k}$ for $k=0$ reaches the desired contact force $F^{d}$ and therefore an initial grasp is performed. Once the object is grasped by the fingertips, the manipulation starts, this is an iterative process that is repeated while a stop signal is not activated or the grasp quality worsens. In each loop, the first step is the computation of the pressing force $F_{k}$ and the absolute positions of the contact points $P_{1_{k}}$ and $P_{2_{k}}$ using the finger direct kinematics described in Section III, the sensor measurements and the knowledge of the sensor geometry. $P_{1_{k}}$ and $P_{2_{k}}$ are expressed in a reference system located at the base of finger $f_{1}$. The next step is the computation of the distance $d_{k}$ between $P_{1_{k}}$ and $P_{2_{k}}$. This distance is used as input parameter to compute the expected contact points $P_{1_{k+1}}$ and $P_{2_{k+1}}$. Besides, adjustments of $d_{k}$ allows the control of the grasping force applied on the object. Then, the expected distance $d_{k+1}$ between contact points is computed as,

$$
d_{k+1}=d_{k}+\Delta d
$$

with $\Delta d$ being a function of the force $F_{k}$ according to the follow relationship,

$$
\Delta d= \begin{cases}0 & \text { if } F_{\min }<F_{k}<F_{\max } \\ +\lambda & \text { if } F_{k} \leq F_{\min } \\ -\lambda & \text { if } F_{k} \geq F_{\max }\end{cases}
$$

where the constant values $F_{\min }, F_{\max }$ and $\lambda$ were empirically defined after experimenting with real objects.

In the manipulation strategy, one of the fingers (independent finger) moves towards the comfort position while the other finger (follower finger) accommodates to the movement of the first one in order to avoid the object falling. The fingers alternate their roles in order to balance the hand movements, making that both fingers advance to the comfort position. The variation $\Delta \theta$ in the finger joints in each step must be small in order to avoid object falls. The most comfortable position of the hand SDH2 is when all joint values are set to 0 degrees. So, in each step the independent finger moves its joints towards 0 value, i.e. following the gradient of the quality measure is equivalent to move the joints to 0 degrees. Then, the next step is the computation of the joints to properly move the independent finger.

Thus, the new joints values $\theta_{2_{k+1}}$ and $\theta_{3_{k+1}}$ of finger $f_{1}$ are given by

$$
\begin{aligned}
& \theta_{2_{k+1}}=\theta_{2_{k}}+\Delta \theta \\
& \theta_{3_{k+1}}=\theta_{3_{k}}+\Delta \theta
\end{aligned}
$$

Once computed the new joint values, $P_{1_{k+1}}$ is computed using direct kinematics. The movement from $P_{1_{k}}$ to $P_{1_{k+1}}$ will produce a change on the object inclination $\Delta \alpha_{k+1}$, given by,

$$
\begin{aligned}
\Delta \alpha_{k+1} & =\arctan \left(\frac{P_{2_{z_{k}}}-P_{1_{z_{k+1}}}}{P_{2_{x_{k}}}-P_{1_{x_{k+1}}}}\right) \\
& -\arctan \left(\frac{P_{2_{z_{k}}}-P_{1_{z_{k}}}}{P_{2_{x_{k}}}-P_{1_{x_{k}}}}\right)
\end{aligned}
$$

The expected contact point $P_{2_{k+1}}$ is computed as a point on a circumference of diameter $d_{k+1}$, which is measured from the contact point $P_{1_{k+1}}, P_{2_{k+1}}$ changes the object inclination in $\Delta \alpha_{k+1}$ (See [12] for a detailed description). Finally, the joint values for finger $f_{2}$ are computed using the inverse kinematics. A similar procedure is applied when $f_{2}$ is the independent finger.

The angles $\psi_{1}$ and $\psi_{2}$ used to verify the friction constraints are computed using the expected contact points. If the friction constraints at $P_{1_{k+1}}$ and $P_{2_{k+1}}$ are satisfied (Eq. 12), and the points $P_{1_{k+1}}$ and $P_{2_{k+1}}$ lie inside the finger workspaces, then, the fingers $f_{1}$ and $f_{2}$ are moved such that the contact points on the fingertips currently at $P_{1_{k}}$ and $P_{2_{k}}$ move to $P_{1_{k+1}}$ and $P_{2_{k+1}}$ respectively, and a new iteration is started; else the hand cannot move to the desired position and the manipulation ends.

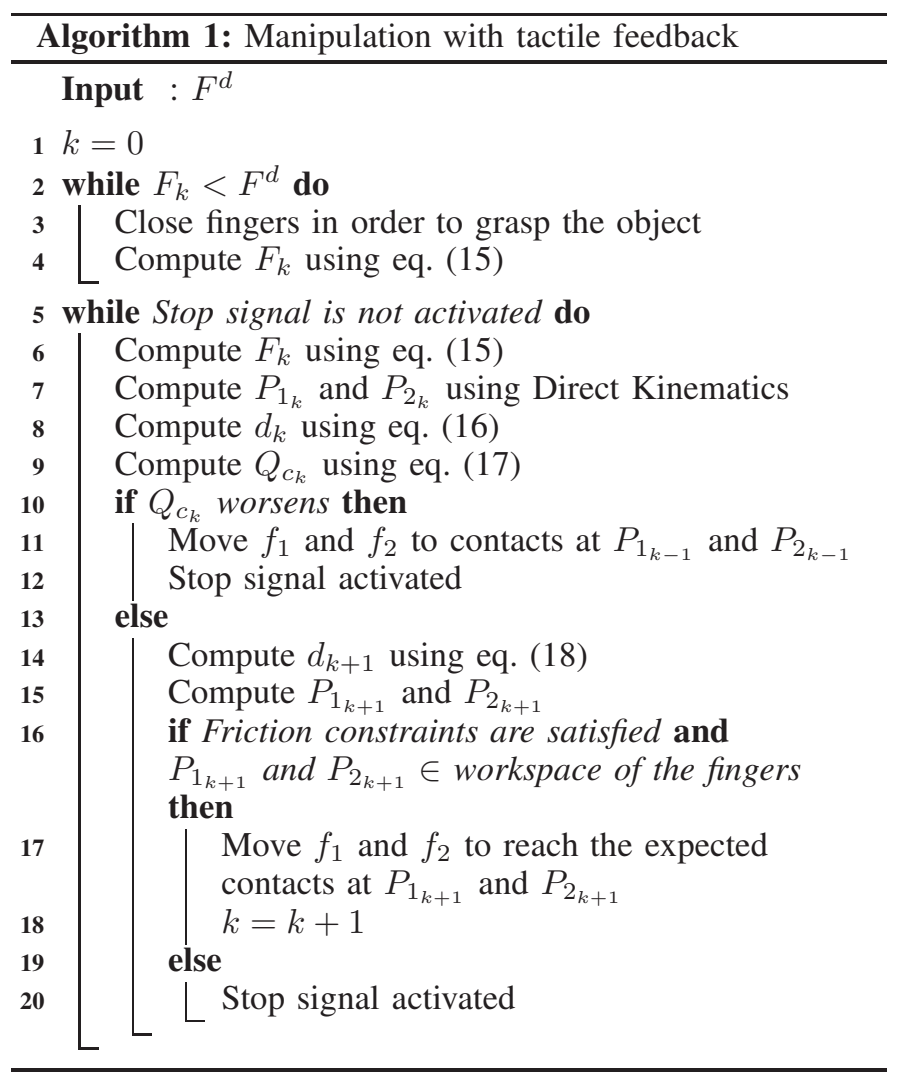

\section{EXPERIMENTAL RESULTS}

In order to illustrate the performance of the proposed approach, the results of manipulating three different objects are presented (see Figure 7). Each object is held between two fingers of the SDH2, then the fingers are closed until the detected contact forces reach the desired value (note that the initial contact points are unknown). After this, the object is 

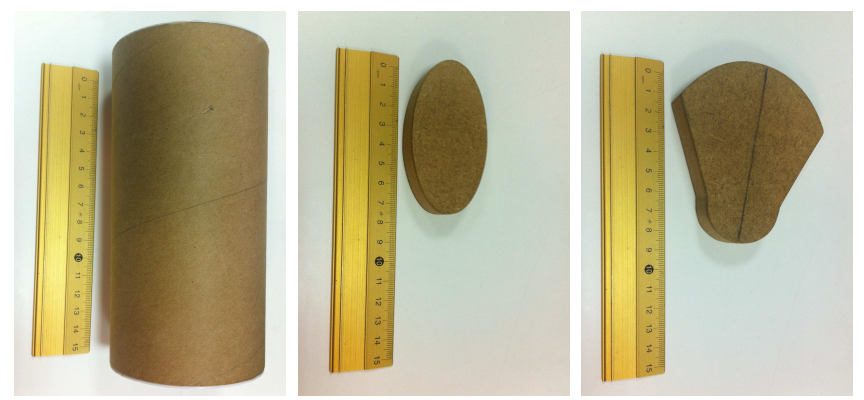

Fig. 7. Three manipulated objects: cylinder, ellipsoid and two-curvatures shape.

manipulated by the two fingers. The material of the surface of the fingertips is rubber and the material of the objects is wood or cardboard, thus we consider a worst case friction coefficient $\mu=0.4$, which is lower than the friction coefficient of between rubber and wood $\mu=0.7$, and rubber and cardboard $\mu=0.5$ [18].

The constant $\lambda$ to adjust the distance between contact points is set to $1 \mathrm{~mm}$, and the desired grasp force $F^{d}$ is set to $20 \mu N$, both values were determined empirically. The variation of the finger joints in each manipulation step was set to 0.5 degrees.

Figure 11 shows snapshots of the manipulation of the cylindrical object (Shown in Figure 7(left)). The hand starts in a wide open configuration to be able to grasp a wide set of objects. The object is grasped by closing the finger of the hand. The initial grasp configuration changes at each execution of the experiment. Then, the manipulation starts and the hands moves to a more comfortable configuration. Figure 8 shows the obtained comfort quality index for the manipulation of the cylindrical object. Note that comfort quality index decreases in each manipulation step, which is an indicator of an improvement in the grasp quality. Figure 9 shows the joints of this hand for the manipulation of the same object, which start far from the center of the joint range and evolve towards the center of the range ( 0 degrees) in order to improve the grasp quality.

Figure 12 shows snapshots of the manipulation of the elliptical object in Figure 7(center). Figure 10 shows the quality index for the manipulation. The evolution of the joints during the manipulation are shown in Figure 14. The behavior of the joints is similar to the obtained when a cylindrical object is manipulated.

Similar results are obtained for the manipulation of a twocurvatures object. Figure 13 shows snapshots of the manipulation of the object in Figure 7(right). Figure 15 shows the variation of the quality index and Figure 16 shows the evolution of the joints.

\section{CONCLUSiOn AND Future WORK}

An approach to get comfortable hand configurations while manipulating unknown objects based on tactile information and force feedback was presented. The experimental results showed that the approach is effective to improve the grasp quality for different objects.

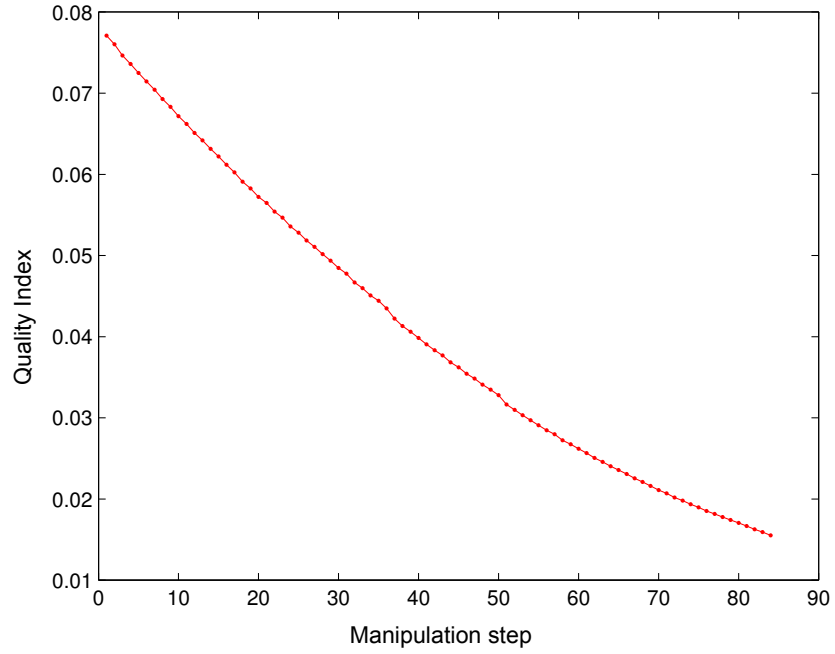

Fig. 8. Quality index $Q_{c}$ for the cylindrical object.

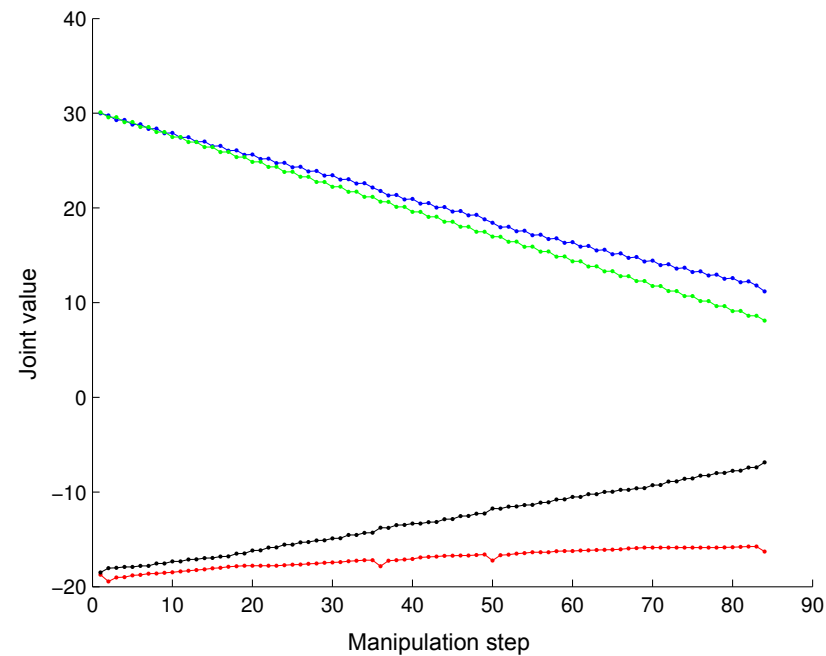

Fig. 9. Obtained joints in the manipulation of the cylindrical object. $\theta_{2}$ in red, $\theta_{3}$ in blue, $\theta_{5}$ in black and, $\theta_{6}$ in green

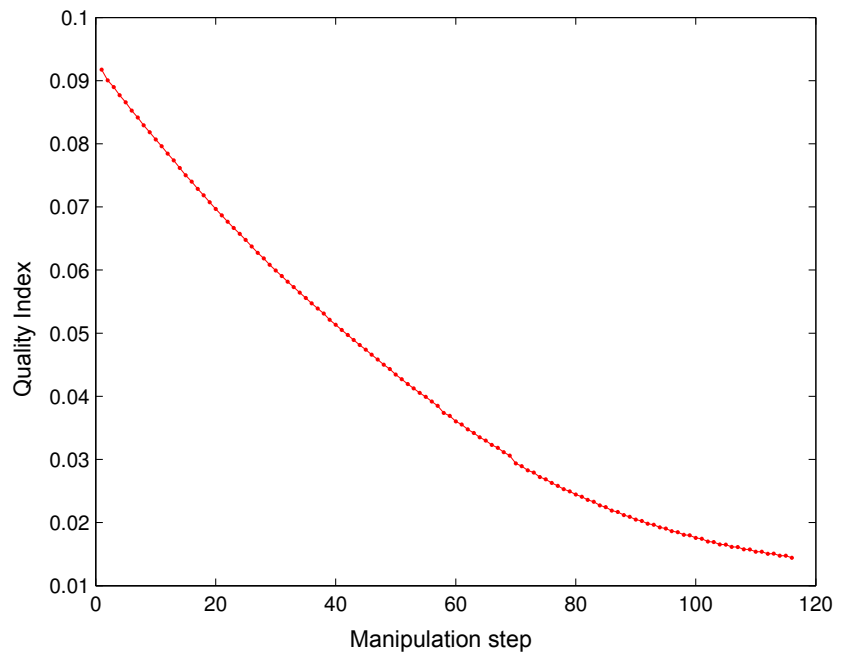

Fig. 10. Quality index $Q_{c}$ for the elliptical object. 


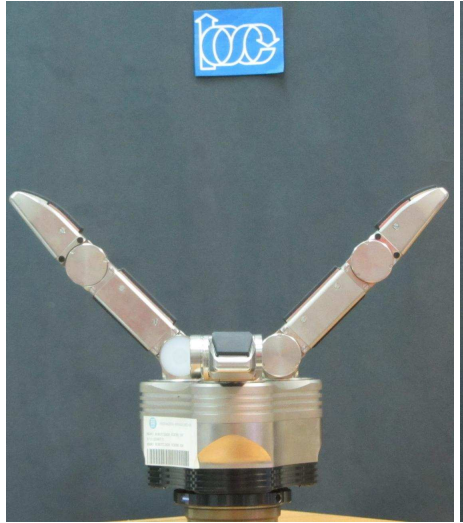

a)

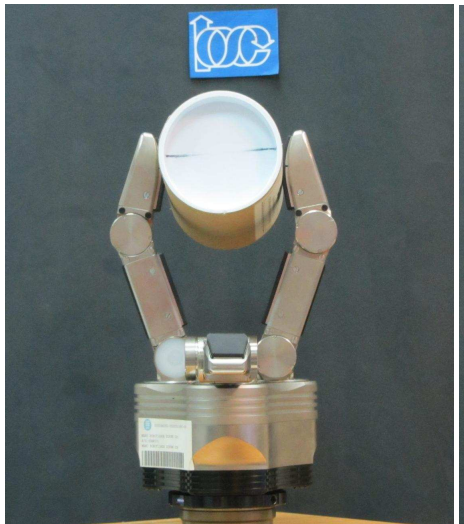

b)

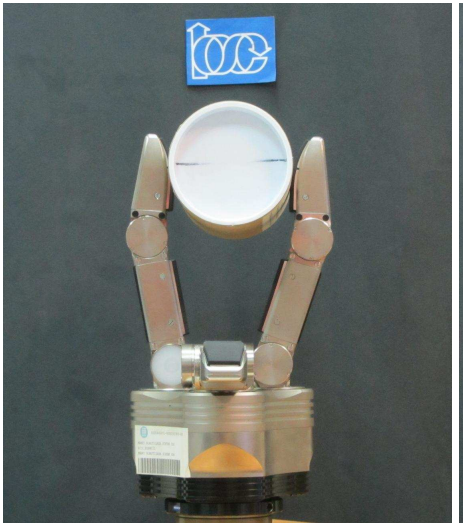

c)

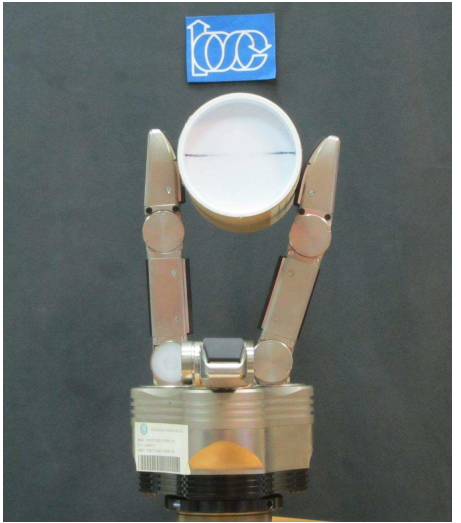

d)

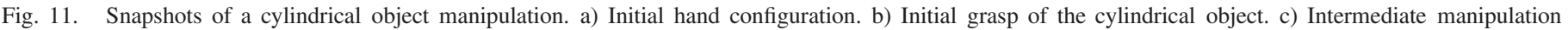
step. d) Final hand configuration with the best comfort quality.

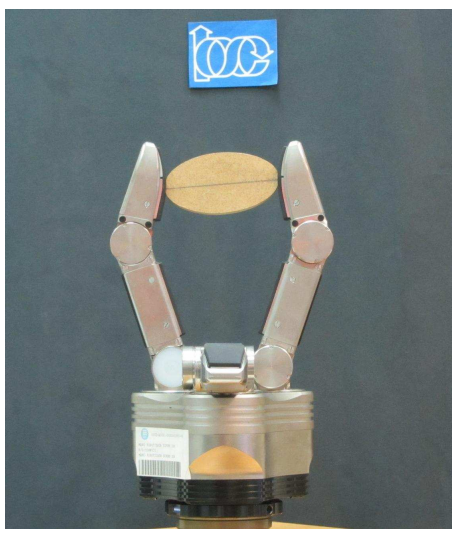

a)

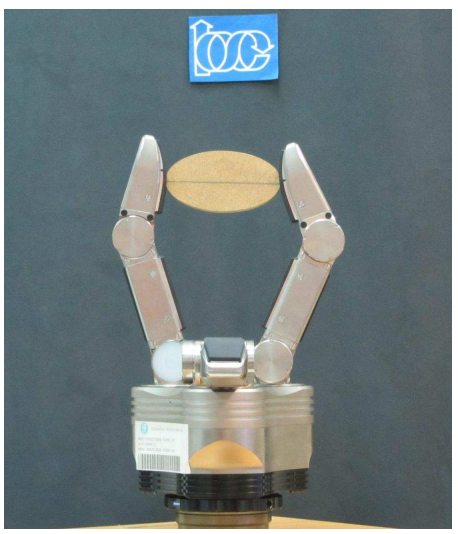

b)

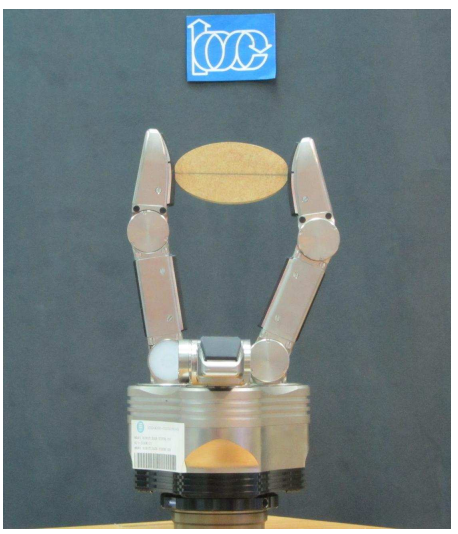

c)

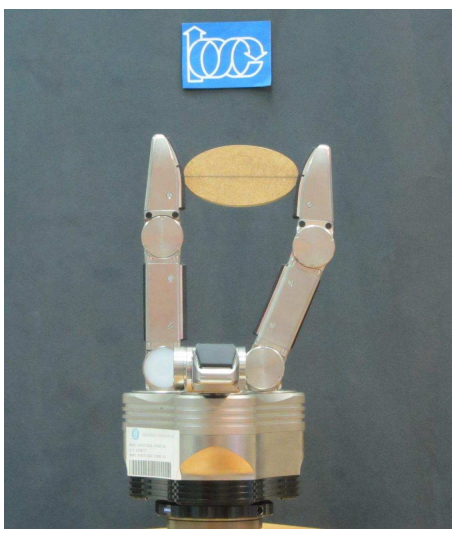

d)

Fig. 12. Snapshots of a elliptical object manipulation. a) Initial grasp. b)-c) Intermediate manipulation step. d) Final hand configuration.

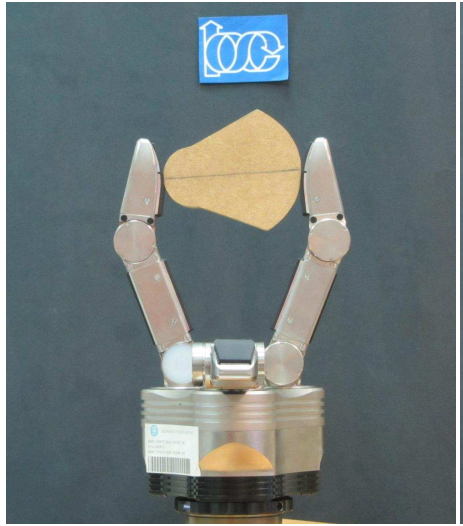

a)

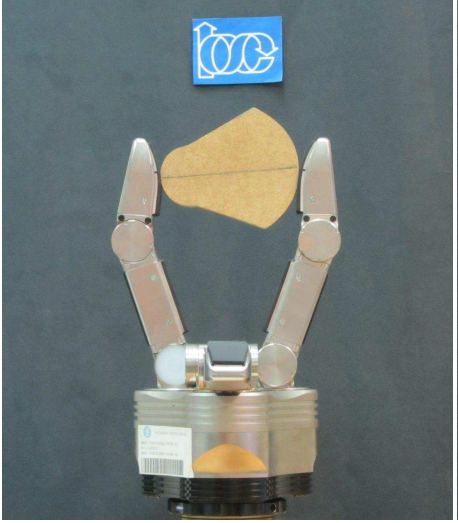

b)

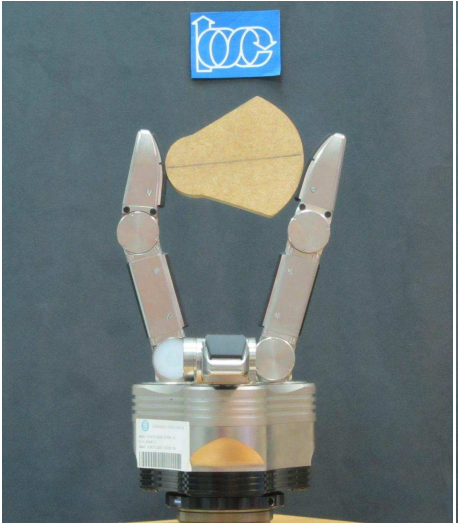

c)

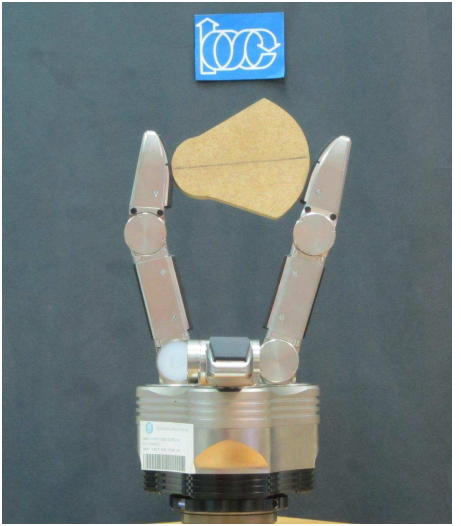

d)

Fig. 13. Snapshots of a cylindrical object manipulation. a) Initial grasp. b)-c) Intermediate manipulation step. d) Final hand configuration. 


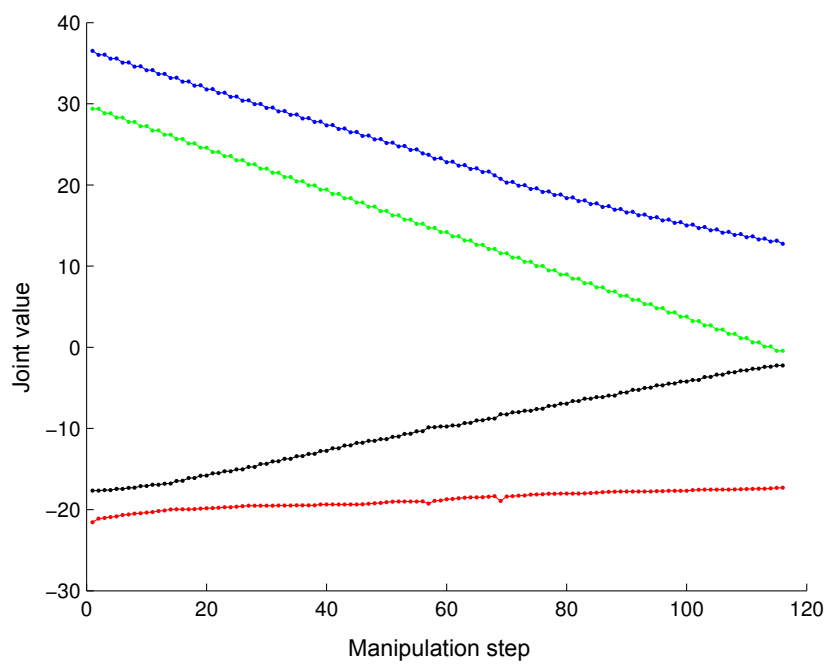

Fig. 14. Obtained joints in the manipulation of the ellipsoid object. $\theta_{2}$ in red, $\theta_{3}$ in blue, $\theta_{5}$ in black and, $\theta_{6}$ in green.

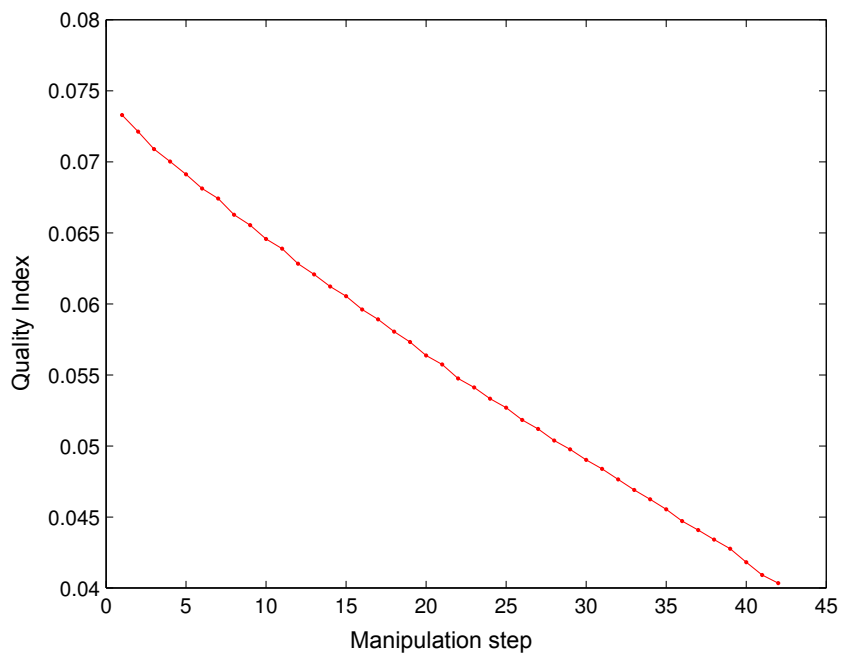

Fig. 15. Quality index $Q_{c}$ for the two shape object.

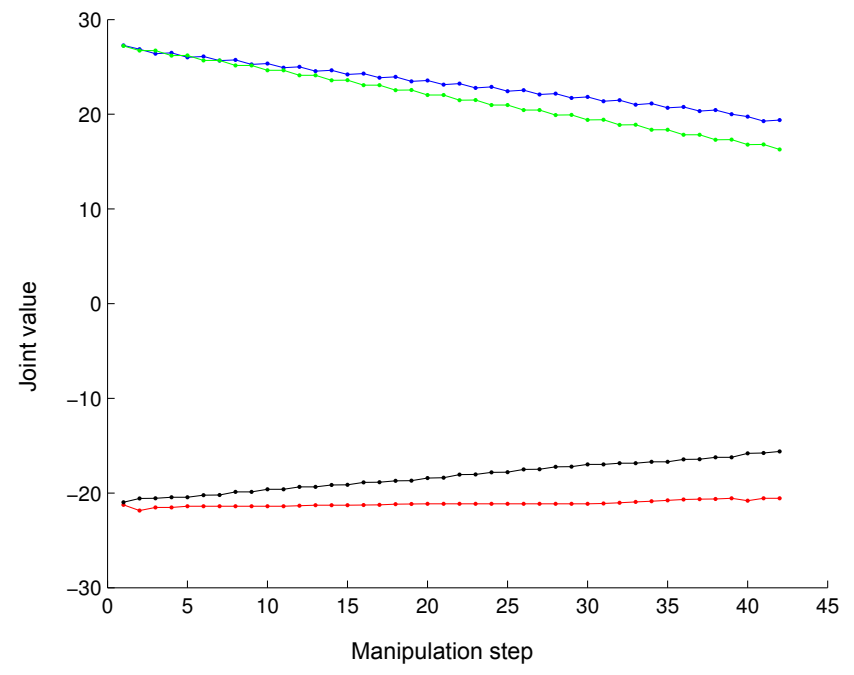

Fig. 16. Obtained joints in the manipulation of the two shape object. $\theta_{2}$ in red, $\theta_{3}$ in blue, $\theta_{5}$ in black and, $\theta_{6}$ in green.
An extension of the implemented work is the inclusion of other grasp quality measures or a combination of them, which consider different grasp characteristics. The gradient of the comfort quality measure is well known, since the more comfortable configuration is in the center of the hand joint range, but with other grasp qualities the gradient must be computed at same time that the manipulation is performed.

\section{REFERENCES}

[1] R. Ma and A. Dollar, "On dexterity and dexterous manipulation," in Proc. IEEE Int. Conf. Advanced Robotics, 2011, pp. 1-7.

[2] H. Iwata and S. Sugano, "Design of anthropomorphic dexterous hand with passive joints and sensitive soft skins," in IEEE/SICE Int. Symp. System Integration, 2009, pp. 129-134.

[3] L. Biagiotti, F. Lotti, C. Melchiorri, and G. Vassura, "How far is the human hand? a review on anthropomorphic robotic end-effectors," University of Bologna, Tech. Rep., 2004.

[4] X. H. Gao, M. Jin, L. Jiang, Z. Xie, P. He, L. Yang, Y. W. Liu, R. Wei, H. G. Cai, H. Liu, J. Butterfass, M. Grebenstein, N. Seitz, and G. Hirzinger, "The hit/dlr dexterous hand: work in progress," in Proc. IEEE Int. Conf. Robotics and Automation, vol. 3, Sept 2003, pp. 3164-3168.

[5] A. Bicchi, "Hands for dexterous manipulation and robust grasping: A difficult road toward simplicity," IEEE Trans. Robotics and Automation, vol. 16, no. 6, pp. 652-662, 2000.

[6] S. Jacobsen, J. Wood, D. Knutti, and K. Biggers, "The utah/m.i.t. dextrous hand: Work in progress," Int. J. Robotics Research, vol. 3, no. 4, pp. 21-50, 1984.

[7] M. Meier, M. Schopfer, R. Haschke, and H. Ritter, "A probabilistic approach to tactile shape reconstruction," IEEE Trans. Robotics, vol. 27, no. 3, pp. 630-635, 2011.

[8] J. Bimbo, S. Rodriguez-Jimenez, H. Liu, X. Song, N. Burrus, L. Senerivatne, M. Abderrahim, and K. Althoefer, "Object pose estimation and tracking by fusing visual and tactile information," in Multisensor Fusion and Integration for Intelligent Systems (MFI), 2012 IEEE Conference on, 2012, pp. 65-70.

[9] V. A. Ho, T. Nagatani, A. Noda, and S. Hirai, "What can be inferred from a tactile arrayed sensor in autonomous in-hand manipulation?" in Automation Science and Engineering (CASE), 2012 IEEE International Conference on, 2012, pp. 461-468.

[10] Y. Bekiroglu, R. Detry, and D. Kragic, "Learning tactile characterizations of object- and pose-specific grasps," in Intelligent Robots and Systems (IROS), 2011 IEEE/RSJ International Conference on, 2011, pp. 1554-1560.

[11] H. Dang, J. Weisz, and P. Allen, "Blind grasping: Stable robotic grasping using tactile feedback and hand kinematics," in Robotics and Automation (ICRA), 2011 IEEE International Conference on, 2011, pp. 5917-5922.

[12] A. Montaño and R. Suárez, "Object shape reconstruction based on the object manipulation," in Proc. IEEE Int. Conf. Advanced Robotics, 2013, pp. 1-6.

[13] C. MacKenzie and T. Iberall, The Grasping Hand, ser. Advances in Psychology. Elsevier Science, 1994.

[14] J. J. Craig, Introduction to Robotics: Mechanics and Control, 2nd ed. Boston, MA, USA: Addison-Wesley Longman Publishing Co., Inc., 1989.

[15] K. Shimoga, "Robot grasp synthesis algorithms: A survey," The International Journal of Robotics Research, vol. 15, no. 3, pp. 230-266, 1996.

[16] R. Suárez, M. Roa, and J. Cornellà, "Grasp quality measures," Polytechnic University of Catalonia, Tech. Rep., 2006.

[17] M. Roa, R. Suárez, and J. Cornellà, "Medidas de calidad para la prensión de objetos," Rev. Iberoamericana de Automática e Informática Industrial, vol. 5, no. 1, pp. 66-82, 2008. [Online]. Available: http://recyt.fecyt.es/index.php/RIAII/article/view/231

[18] M. Kutz, Mechanical Engineers' Handbook, ser. A WileyInterscience publication. Wiley, 1998. [Online]. Available: http://books.google.es/books?id=65KyGC6kvqIC 